PROCEEDINGS OF THE

AMERICAN MATHEMATICAL SOCIETY

Volume 135, Number 7, July 2007, Pages 1949-1957

S 0002-9939(07)08222-6

Article electronically published on February 16, 2007

\title{
BOUNDS ON THE CASTELNUOVO-MUMFORD REGULARITY OF TENSOR PRODUCTS
}

\author{
GIULIO CAVIGLIA
}

(Communicated by Bernd Ulrich)

\begin{abstract}
In this paper we show how, given a complex of graded modules and knowing some partial Castelnuovo-Mumford regularities for all the modules in the complex and for all the positive homologies, it is possible to get a bound on the regularity of the zero homology. We use this to prove that if $\operatorname{dim} \operatorname{Tor}_{1}^{R}(M, N) \leq 1$, then $\operatorname{reg}(M \otimes N) \leq \operatorname{reg}(M)+\operatorname{reg}(N)$, generalizing results of Chandler, Conca and Herzog, and Sidman. Finally we give a description of the regularity of a module in terms of the postulation numbers of filter regular hyperplane restrictions.
\end{abstract}

\section{INTRODUCTION}

Let $R=K\left[X_{1}, \ldots, X_{n}\right]$ be a polynomial ring over a field $K$, let $M$ be a finitely generated graded $R$-module and let $I \subset R$ be an ideal. Recently some work has been done to study when the Castelnuovo-Mumford regularity of $I^{r}$ can be bounded by $r$ times the regularity of $I$, and more generally when the regularity of $I M$ can be bounded by the sum of the regularity of $I$ and $M$. This is not always the case; see the papers of Sturmfels $[\mathrm{St}$ and Conca, Herzog [CH] for counterexamples. On the other hand, under the hypothesis that $\operatorname{dim}(R / I) \leq 1$, Chandler $\mathrm{Ch}$ and Geramita, Gimigliano and Pitteloud GGP showed that $\operatorname{reg}\left(I^{r}\right) \leq r \operatorname{reg}(I)$. In a recent paper Conca and Herzog $[\mathrm{CH}$ proved, using similar methods to the one in $\mathrm{Ch}$, that under the same assumption (i.e. $\operatorname{dim}(R / I) \leq 1) \operatorname{reg}(I M) \leq \operatorname{reg}(I)+\operatorname{reg}(M)$. An extension of the latter was recently done by Sidman [Si who showed that if two ideals of $R$, say $I$ and $J$, define schemes whose intersection is a finite set of points, then $\operatorname{reg}(I J) \leq \operatorname{reg}(I)+\operatorname{reg}(J)$. She deduced this theorem from a result in the same paper [Si] which bounded the regularity of a tensor product of sheaves.

In the first section of this paper we show how the same technique as in [Si] can be applied to prove a stronger statement, i.e. that given $M$ and $N$ graded $R$-modules such that $\operatorname{dim} \operatorname{Tor}_{1}^{R}(M, N) \leq 1$, then $\operatorname{reg}(M \otimes N) \leq \operatorname{reg}(M)+\operatorname{reg}(N)$. It is easy to see that this implies all the other results mentioned above. This theorem has been recently applied by Daniel Giaimo Gi to prove the Eisenbud-Goto regularity conjecture for connected absolutely reduced curves.

Received by the editors March 3, 2003 and, in revised form, February 1, 2005.

2000 Mathematics Subject Classification. Primary 13D45, 13D02.

Key words and phrases. Castelnuovo-Mumford regularity, postulation number, filter-regular sequence.

The author was partially supported by the "Istituto Nazionale di Alta Matematica Francesco Severi", Rome.

(C)2007 American Mathematical Society Reverts to public domain 28 years from publication 
In section 2 we deduce from a formula of Serre that the Castelnuovo-Mumford regularity can be described in terms of the postulation numbers of filter regular hyperplane restrictions, where the postulation number $\alpha(M)$ of a module $M$ is defined as the largest nonnegative integer for which the Hilbert function of $M$ is not equal to the corresponding Hilbert polynomial. More precisely we show that given a finitely generated graded $R$-module $M$ with $\operatorname{dim}(M)=d$, we have

$$
\operatorname{reg}(M)=\max _{i \in\{0, \ldots, d\}}\left\{\alpha\left(M /\left(l_{1}, \ldots, l_{i}\right) M\right)-\sum_{j=1}^{i}\left(D_{j}-1\right)\right\},
$$

where $l_{1}, \ldots, l_{d}$ is a filter regular sequence on $M$ of degrees $D_{1}, \ldots, D_{d}$.

We thank the referee for pointing our attention to the first section of a paper of Malgrange [Ma. Looking at another definition of regularity that goes back to E. Cartan (1901), for which Janet (1927) proved that $m$-regularity implies $(m+1)$ regularity, the above theorem, at least for linear forms, follows from the equivalence to today's definition (attributed by Malgrange to Quillen, Serre and Mumford in the 1960's). Moreover the extension to higher degree forms may also be done along the lines of $[\mathrm{Ma}]$.

\section{Generalities}

From now on by $R$ we denote the polynomial ring $K\left[X_{1}, \ldots, X_{n}\right]$ and by $R_{+}$ its homogeneous maximal ideal. Given a graded $R$-module $M=\bigoplus_{i \in \mathbb{Z}} M_{i}$, we will denote $\bigoplus_{i \in \mathbb{Z}, i>a} M_{i}$ by $M_{>a}$. We want to define some partial Castelnuovo-Mumford regularities for $M$ with respect to a set of indices $\mathcal{X} \subseteq \mathbb{N}$ as follows.

Definition 2.1. Given a set of indices $\mathcal{X} \subseteq \mathbb{N}$ and a finitely generated graded $R$-module $M$, we say that $M$ is $m$-regular with respect to $\mathcal{X}$ (i.e. $m$-reg ${ }^{\mathcal{X}}$ ) if we have $H_{R_{+}}^{i}(M)_{>m-i}=0$ for all $i \in \mathcal{X}$. The regularity of $M$ with respect to $\mathcal{X}$ is defined to be the minimum of all the $m$ for which $M$ is $m$-reg ${ }^{\mathcal{X}}$.

Remark 2.2. We should observe that, from the Grothendieck vanishing theorem, all the local cohomology modules are zero for indexes bigger than $n$. Note also that when $\mathcal{X}=\{0, \ldots, n\}$, the $m$-reg ${ }^{\mathcal{X}}$ agrees with $m$-regularity in the sense of Castelnuovo-Mumford.

The next lemma describes the behavior of the regularity with respect to $\mathcal{X}$ for exact sequences. We will use the following notation: given $a \in \mathbb{Z}$ we set $\mathcal{X}+a$ to be $\{i+a \mid i \in \mathcal{X}\} \cap \mathbb{N}$.

Lemma 2.3. Given a short exact sequence of finitely generated graded R-modules,

$$
0 \longrightarrow \mathrm{M} \longrightarrow \mathrm{N} \longrightarrow \mathrm{P} \longrightarrow 0,
$$

we have:

(1) If $M$ and $P$ are $m$-reg ${ }^{\mathcal{X}}$ so is $N$.

(2) If $N$ is $m$-reg ${ }^{\mathcal{X}}$ and $P$ is $(m-1)$-reg ${ }^{\mathcal{X}-1}$, then $M$ is $m$-reg ${ }^{\mathcal{X}}$.

(3) If $M$ is $(m+1)-\operatorname{reg}^{\mathcal{X}+1}$ and $N$ is $m$-reg ${ }^{\mathcal{X}}$, then $P$ is $m$-reg ${ }^{\mathcal{X}}$.

Proof. The result follows from the long exact sequence of local cohomology modules. 


\section{Regularity of tensor products And Hom of modules}

The following lemma was inspired by Lemma 1.4 in [Si].

Lemma 3.1. Let $\mathbf{C}$ be a complex of finitely generated graded $R$-modules

$$
\mathbf{C}: 0 \longrightarrow C_{n} \longrightarrow C_{n-1} \longrightarrow C_{0} \longrightarrow 0
$$

If $C_{i}$ is $(m+i)-$ reg $^{\mathcal{X}+i}$ for all $i>0$ and the $i^{\text {th }}$ homology $H_{i}(\mathbf{C})$ is $(m+i+1)$ $\operatorname{reg}^{\mathcal{X}+i+1}$ for all $i>0$, then:

(1) The $i^{\text {th }}$ boundary $B_{i}$ is $(m+i+1)-\mathrm{reg}^{\mathcal{X}+i+1}$ for all $i \geq 0$.

(2) If $C_{0}$ is $m-\mathrm{reg}^{\mathcal{X}}$, then so is $H_{0}(\mathbf{C})$.

If $C_{n-i}$ is $(m-i)-\operatorname{reg}^{\mathcal{X}-i}$ for all $i \geq 0$ and the $(n-i)^{\text {th }}$ homology $H_{n-i}(\mathbf{C})$ is $(m-i-1)-\mathrm{reg}^{\mathcal{X}-i-1}$ for all $i>0$, then:

$\left(1^{\prime}\right)$ The $(n-i)^{\text {th }}$ cycles $Z_{n-i}$ are $(m-i)-\operatorname{reg}^{\mathcal{X}-i}$ for all $i \geq 0$.

$\left(2^{\prime}\right)$ In particular $H_{n}(\mathbf{C})$ is $m-\mathrm{reg}^{\mathcal{X}}$.

Proof. First we prove (1). Note that when $i=n$ the boundary $B_{i}=B_{n}=0$ is trivially $(m+i+1)-$ reg $^{\mathcal{X}+i+1}$. We can therefore do a reverse induction on $i$. Consider the following diagram with exact rows and columns:

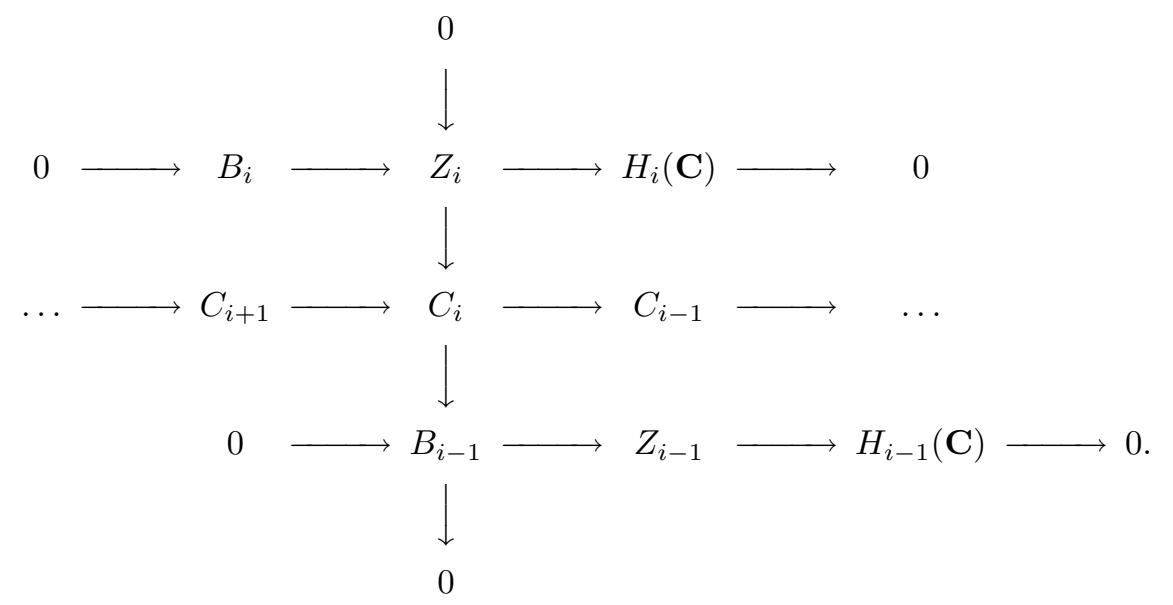

By induction we know that $B_{i}$ is $(m+i+1)-\operatorname{reg}^{\mathcal{X}+i+1}$ and by assumption $H_{i}(\mathbf{C})$ is $(m+i+1)-$ reg $^{\mathcal{X}+i+1}$ so, applying Lemma 2.3 to the top exact row in the diagram above, we deduce that $Z_{i}$ is $(m+i+1)-$ reg $^{\mathcal{X}+i+1}$. Now, since $C_{i}$ is $(m+i)-$ reg $^{\mathcal{X}+i}$, applying Lemma 2.3 to the exact column of the diagram we obtain that $B_{i-1}$ is $(m+i)-\operatorname{reg}^{\mathcal{X}+i}$; this completes the induction and proves $(1)$.

We now prove (2). Consider the exact sequence

$$
0 \longrightarrow B_{0} \longrightarrow C_{0} \longrightarrow H_{0} \longrightarrow 0 \text {. }
$$

By part (1) we know that $B_{0}$ is $(m+1)-\operatorname{reg}^{\mathcal{X}+1}$ and by assumption $C_{0}$ is $m$-reg ${ }^{\mathcal{X}}$. Therefore from Lemma 2.3 it follows that $H_{0}$ is $m$-reg ${ }^{\mathcal{X}}$.

The proofs of $\left(1^{\prime}\right)$ and $\left(2^{\prime}\right)$ follow similar lines. Note that since $Z_{n} \cong H_{n}(\mathbf{C})$, it is sufficient to prove $\left(1^{\prime}\right)$. Moreover $Z_{0}=C_{0}$ is $(m-n)-$ reg $^{\mathcal{X}-n}$; we can therefore do a reverse induction on $i$. Apply Lemma 2.3 (2) to the last row in the diagram to get $B_{n-i}$ is $(m-i)-\mathrm{reg}^{\mathcal{X}-i}$ and then apply Lemma 2.3 (2) to the exact column to get $Z_{n-i+1}$ is $(m-i+1)-\operatorname{reg}^{\mathcal{X}-i+1}$. This completes the induction. 
3.1. Bounds on the regularity of the tensor product. An easy corollary of Lemma 3.1(2) is the following.

Theorem 3.2. Let $M$ and $N$ be finitely generated graded $R$-modules such that $\mathcal{X}=\{a, \ldots, n\}$ for $a \geq 0, M$ is $m$-regular (i.e. $m$-reg $\left.{ }^{\{0, \ldots, n\}}\right), N$ is $s$-reg ${ }^{\mathcal{X}}$ and $\operatorname{Tor}_{i}^{R}(M, N)$ is $(m+s+i+1)-\operatorname{reg}^{\mathcal{X}+i+1}$ for all $i>0$. Then $M \otimes_{R} N$ is $(m+s)-\operatorname{reg}^{\mathcal{X}}$.

Proof. Take a minimal graded free resolution $\mathbb{F}: \cdots \rightarrow F_{i} \rightarrow \cdots \rightarrow F_{0}$ of $M$. Note that since $M$ is $m$-regular, the lowest possible shift appearing in $F_{i}$ is $-m-i$. Hence $F_{i} \otimes N$ is $(m+s+i)-$ reg $^{\mathcal{X}}$ and so in particular it is $(m+s+i)$-reg ${ }^{\mathcal{X}+i}$. The homologies of the complex $\mathbb{F} \otimes_{R} N$ are $\operatorname{Tor}_{i}^{R}(M, N)$, and by assumption they are $(m+s+i+1)-\operatorname{reg}^{\mathbb{X}+i+1}$, for $i>0$. The conclusion follows from Lemma 3.1 (2) applied to $\mathbb{F} \otimes N$ after noting that $H_{0}(\mathbb{F} \otimes N)$ is $M \otimes N$.

Remark 3.3. Note that the condition, " $\operatorname{Tor}_{i}^{R}(M, N)$ is $(m+s+i+1)-$ reg $^{\mathcal{X}+i+1}$ " of Theorem 3.2 is clearly satisfied when the Krull dimension of $\operatorname{Tor}_{i}^{R}(M, N)$ is less than or equal to the minimum of $\mathcal{X}+i$ (since the relevant local cohomology modules are zero for reasons of dimension).

Setting $\mathcal{X}=\{0, \ldots, n\}$, and noting that by rigidity of Tor (see $\mathrm{Au}$. Theorem 2.1) $\operatorname{dim} \operatorname{Tor}_{1}^{R}(M, N) \leq 1$ is equivalent to $\operatorname{dim} \operatorname{Tor}_{i}^{R}(M, N) \leq 1$ for all $i \geq 1$, we have the following corollary.

Corollary 3.4. Let $M$ be an $m$-regular finitely generated graded $R$-module and let $N$ be an $n$-regular finitely generated graded $R$-module such that $\operatorname{dim} \operatorname{Tor}_{1}^{R}(M, N) \leq$ 1. Then $M \otimes N$ is $(m+n)$-regular.

From Corollary 3.4 we can deduce:

Theorem 3.5. Let $I \subseteq R$ be a homogeneous ideal and let $M$ be a finitely generated graded $R$-module such that the dimension of $\operatorname{Tor}_{1}^{R}(M, R / I)$ is less than or equal to 1. Then $\operatorname{reg}(I M) \leq \operatorname{reg}(I)+\operatorname{reg}(M)$.

Proof. First note that unless $I$ is the whole ring (in which case the result is obvious), we can assume that $\operatorname{reg}(I)>0$. From the exact sequence

$$
0 \longrightarrow I \longrightarrow R \longrightarrow R / I \longrightarrow 0
$$

we get $\operatorname{reg}(R / I)=\operatorname{reg}(I)-1$. By Corollary $3.4 \operatorname{reg}(M / I M)=\operatorname{reg}\left(M \otimes_{R} R / I\right) \leq$ $\operatorname{reg}(M)+\operatorname{reg}(I)-1$. On the other hand applying Lemma 2.3(2) to the exact sequence

$$
0 \longrightarrow I M \longrightarrow M \longrightarrow M / I M \longrightarrow 0,
$$

we obtain $\operatorname{reg}(I M) \leq \max \{\operatorname{reg}(M), \operatorname{reg}(M / I M)+1\}$ which is less than or equal to

$$
\max \{\operatorname{reg}(M), \operatorname{reg}(M)+\operatorname{reg}(I)-1+1\} \leq \operatorname{reg}(M)+\operatorname{reg}(I) .
$$

Theorem 3.5 implies the following.

Theorem 3.6 (Conca, Herzog, Theorem 2.5 of $[\mathrm{CH}]$ ). Let $I \subset R$ be a homogeneous ideal with $\operatorname{dim} R / I \leq 1$ and let $M$ be a finitely generated graded $R$-module. Then $\operatorname{reg}(I M) \leq \operatorname{reg}(I)+\operatorname{reg}(M)$.

Theorem 3.7 (Sidman, Theorem 1.8 of [Si]). Let $I, J$ be homogeneous ideals of $R$ such that the dimension of $R /(I+J)$ is less than or equal to 1 . Then $\operatorname{reg}(I J) \leq$ $\operatorname{reg}(I)+\operatorname{reg}(J)$. 
3.2. Bounding the Castelnuovo-Mumford regularity of $\operatorname{Hom}_{R}(M, N)$. Similar reasoning as in Theorem 3.2 can be used to prove a bound for the regularity of $\operatorname{Hom}_{R}(M, N)$, where $M$ and $N$ are finitely generated graded $R$-modules. In this context the dimensional condition required of $\operatorname{Tor}_{1}^{R}(M, N)$ has an analogue in certain conditions on the depth of $\operatorname{Ext}_{R}^{i}(M, N)$.

We prove the following:

Theorem 3.8. Let $M$ and $N$ be finitely generated graded $R$-modules. Let $m$ be the lowest degree of a homogeneous minimal generator for $M$, and let $\mathcal{X}=$ $\{0, \ldots, a\}, a \leq n$, be a set of indices. If $N$ is $s$-reg $\mathcal{X}$ and $\operatorname{Ext}_{R}^{i}(M, N)$ is $(s-m-$ $i-1)$-reg ${ }^{\mathcal{X}-i-1}$ for all $i>0$, then $\operatorname{Hom}_{R}(M, N)$ is $(s-m)$-reg ${ }^{\mathcal{X}}$.

Proof. Take a minimal graded free resolution $\mathbb{F}: \cdots \rightarrow F_{i} \rightarrow \cdots \rightarrow F_{0}$ of $M$. Note that, since the lowest degree of a homogeneous minimal generator for $M$ is $m$, the biggest possible shift appearing in $F_{i}$ is less than or equal to $-m-i$. Hence $\operatorname{Hom}_{R}\left(F_{i}, N\right)$ is $(s-m-i)-$-reg $^{\mathcal{X}}$, so in particular it is $(s-m-i)$-reg ${ }^{\mathcal{X}-i}$. The homologies of the complex $\operatorname{Hom}_{R}(\mathbb{F}, N)$ are $\operatorname{Ext}_{R}^{i}(M, N)$, and by assumption they are $(s-m-i-1)-\operatorname{reg}^{\mathbb{X}-i-1}$ for all $i>0$. The conclusions follow from Lemma 3.1 $\left(2^{\prime}\right)$ applied to $\operatorname{Hom}_{R}(\mathbb{F}, N)$ after noting that $H_{n}\left(\operatorname{Hom}_{R}(\mathbb{F}, N)\right)$ is $\operatorname{Hom}_{R}(M, N)$.

Remark 3.9. The condition " $\operatorname{Ext}_{R}^{i}(M, N)$ is $(s-m-i-1)$-reg ${ }^{\mathcal{X}-i-1}$ for all $i>0$ "

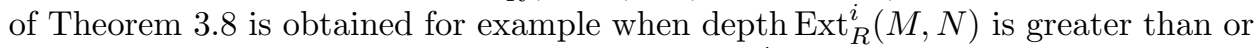
equal to $n-i$ for all $i>0$, because in this case $H_{R_{+}}^{j}\left(\operatorname{Ext}_{R}^{i}(M, N)\right)=0$ for $j<n-$ $i-1$. On the other hand, since for any prime ideal $P$ of ht $P<i, \operatorname{Ext}_{R}^{i}(M, N)_{P}=0$, we have $\operatorname{dim} \operatorname{Ext}_{R}^{i}(M, N) \leq n-i$. Therefore $\operatorname{depth} \operatorname{Ext}_{R}^{i}(M, N) \geq n-i$ if and only if $\operatorname{Ext}_{R}^{i}(M, N)$ is Cohen-Macaulay.

Hence we have the following result and that is analogous to Theorem 3.4 .

Theorem 3.10. Let $M$ be a finitely generated graded $R$-module with $m$ the lowest degree of a homogeneous minimal generator of $M$, and let $N$ be a finitely generated graded $R$-module such that $\operatorname{Ext}_{R}^{i}(M, N)$ is Cohen-Macaulay for all $i>0$. Then $\operatorname{reg}\left(\operatorname{Hom}_{R}(M, N)\right) \leq \operatorname{reg}(N)-m$.

\section{The Castelnuovo-Mumford Regularity in terms of Postulation NUMBERS OF SOME HYPERPLANE SECTIONS}

Let $R$ be $K\left[X_{1}, \ldots, X_{n}\right]$ with the standard grading and let $M=\bigoplus_{i \in \mathbb{Z}} M_{i}$ be a finitely generated graded $R$-module of Krull dimension $d$. In this section we prove how the Castelnuovo-Mumford regularity of $M$ can be obtained as the maximum of all the postulation numbers of $d$ filter regular hyperplane sections. In the following we will denote by $H_{M}(i)$ the value at $i$ of the Hilbert function of $M$ (i.e. $H_{M}(i)=$ $\operatorname{dim}_{K} M_{i}$ ), and with $P_{M}(i)$ the corresponding Hilbert polynomial. It is well known that $P_{M}(i)$ agrees with $H_{M}(i)$ for $i \gg 0$. We recall also that, by a theorem of Hilbert, the Hilbert series (i.e. the formal series defined as $\sum_{i \in \mathbb{Z}} H_{M}(i) Z^{i}$ ) has a rational expression $\frac{h(Z)}{(1-Z)^{d}}$, where $h(Z) \in \mathbb{Z}[Z, 1 / Z]$. When a graded $R$-module $M$ has dimension 0 , we will denote by $\max M$ the degree of its highest nonzero graded component.

Definition 4.1. Let $M$ be a finitely generated graded $R$-module with Hilbert series $\frac{h(Z)}{(1-Z)^{d}}$. Let $h(Z)=\sum_{i=a}^{b} c_{i} Z^{i}$ with $c_{b} \neq 0$. We set the postulation number of $M$ to be $\alpha(M)=b-d$. 
Remark 4.2. It is a well-known fact that the postulation number of $M$ is equal to the highest degree $i$ for which the Hilbert function differs from the Hilbert polynomial (i.e. $H_{M}(i)-P_{M}(i) \neq 0$ ). For a proof see for example Proposition 4.1.12 in $[\mathrm{BH}]$. The following formula of Serre (see $[\overline{\mathrm{BH}}$, Theorem 4.4.3, for a proof),

$$
H_{M}(i)-P_{M}(i)=\sum_{j=0}^{d}(-1)^{j} \operatorname{dim}_{K} H_{R_{+}}^{j}(M)_{i} \text { for all } i \in \mathbb{Z},
$$

shows how the postulation number of $M$ can be defined in terms of the local cohomology modules $H_{R_{+}}^{i}(M)$.

Definition 4.3. A homogeneous element $l \in R$ of degree $D$ is filter regular on a graded $R$-module $M$ if the multiplication map $l: M_{i-D} \rightarrow M_{i}$ is injective for all $i \gg 0$. A sequence $l_{1}, \ldots, l_{m}$ of homogeneous elements of $R$ is called a filter regular sequence on $M$ if $l_{i}$ is filter regular on $M /\left(l_{1}, \ldots, l_{i}-1\right) M$ for $i=1, \ldots, m$.

Remark 4.4. Since $H_{R_{+}}^{0}(M)=\left\{u \in M \mid\left(R_{+}\right)^{k} u=0\right.$ for some $\left.k\right\}$, then $l$ is filter regular on $M$ if and only if $l$ is a nonzerodivisor on $M / H_{R_{+}}^{0}(M)$.

The proposition below will be useful in the sequel. The proof uses a slight modification of the argument in [Mu (pp. 101-102). Moreover, some of its corollaries (Corollary 4.6. Corollary 4.7 and also Theorem 4.11) can also be obtained using the same method as in $[\mathrm{Mu}]$.

Proposition 4.5. Let $M$ be a finitely generated graded $R$-module and let $l \in R$ be a filter regular element on $M$ of degree $D$. Then for any set of indices $\mathcal{X} \subseteq\{0, \ldots, n\}$ we have:

(1) $\operatorname{reg}^{\mathcal{X}+1}(M) \leq \operatorname{reg}^{\mathcal{X} \cup(\mathcal{X}+1)}(M / l M)-D+1$,

(2) $\operatorname{reg}^{\mathcal{X}}(M / l M)-D+1 \leq \operatorname{reg}^{\mathcal{X}} \cup(\mathcal{X}+1)(M)$.

Proof. Consider the short exact sequence

$$
0 \longrightarrow M / 0:_{M} l(-d) \stackrel{\cdot l}{\longrightarrow} M \longrightarrow M / l M \longrightarrow 0 .
$$

Note that, since $l$ is filter regular on $M, H_{R_{+}}^{i}\left(M / 0:_{M} l(-D)\right) \cong H_{R_{+}}^{i}(M)(-D)$ for all $i>0$. Looking at the long exact sequence of local cohomology modules, we have

$$
\begin{gathered}
\ldots H_{R_{+}}^{i}(M) \longrightarrow H_{R_{+}}^{i}(M / l M) \longrightarrow H_{R_{+}}^{i+1}(M)(-D) \longrightarrow \\
\longrightarrow H_{R_{+}}^{i+1}(M) \longrightarrow H_{R_{+}}^{i+1}(M / l M) \longrightarrow
\end{gathered}
$$

for all $i \geq 0$.

Let $j>\operatorname{reg}^{\mathcal{X}}(M / l M)-D+1$ and let $i \in \mathcal{X}$. Consider the exact sequence of $K$-vector spaces given by the graded pieces of degree $j-i+D-1$ of the previous sequence. Because of the choice of $j$, we have $H_{R_{+}}^{i}(M / l M)_{j-i+D-1}=$ $H_{R_{+}}^{i+1}(M / l M)_{j-i+D-1}=0$. Therefore,

$$
H_{R_{+}}^{i+1}(M)(-D)_{j-i+D-1} \cong H_{R_{+}}^{i+1}(M)_{j-i+D-1},
$$

that is,

$$
H_{R_{+}}^{i+1}(M)_{j-i-1} \cong H_{R_{+}}^{i+1}(M)_{j-i+D-1} .
$$

After a simple induction, $H_{R_{+}}^{i+1}(M)_{j-i-1} \cong H_{R_{+}}^{i+1}(M)_{j-i+s D-1}$ for any $s>0$. Since $H_{R_{+}}^{i+1}(M)$ is Artinian, we obtain that $H_{R_{+}}^{i+1}(M)_{j-i-1}=0$ for all $i \in \mathcal{X}$, which implies part (1). 
We now prove part (2). Take $j>\operatorname{reg}^{\mathcal{X} \cup(\mathcal{X}+1)}(M)+D-1$ and $i \in \mathcal{X}$. From the choice of $j$, we have $H_{R_{+}}^{i}(M)_{j-i}=H_{R_{+}}^{i+1}(M)(-D)_{j-i}=0$ for any $i \in \mathcal{X}$. In particular, looking at the $(j-i)^{\text {th }}$ graded component of the long exact sequence of local cohomology modules, we get $H_{R_{+}}^{i}(M / l M)_{j-i}=0$ for all $i \in \mathcal{X}$, which implies part (2).

Proposition 4.5 has the following corollaries:

Corollary 4.6. Given a finitely generated graded $R$-module $M$ and a filter regular element $l$ of degree $D$, we have

$$
\operatorname{reg}\left(M / H_{R_{+}}^{0}(M)\right) \leq \operatorname{reg}(M / l M)-D+1 .
$$

Proof. Set $\mathcal{X}=\{0, \ldots, n\}$ and note that $\operatorname{reg}\left(M / H_{R_{+}}^{0}(M)\right)=\operatorname{reg}^{\mathcal{X}+1}(M)$. The conclusion follows from Proposition 4.5(1).

Corollary 4.7 ( $[\mathrm{CH}]$, Proposition 1.2). Given a finitely generated graded $R$-module $M$ and a filter regular element $l$ of degree $D$, we have

$$
\operatorname{reg}(M)=\max \left\{\max H_{R_{+}}^{0}(M), \operatorname{reg}(M / l M)-D+1\right\} .
$$

Proof. Take $\mathcal{X}=\{0, \ldots, n\}$, and note that $\operatorname{reg}(M)=\max \left\{\operatorname{reg}^{\{0\}}(M), \operatorname{reg}^{\mathcal{X}+1}(M)\right\}$. Clearly $\operatorname{reg}^{\{0\}}(M)=\max H_{R_{+}}^{0}(M)$. From Proposition 4.5)(1) we have $\operatorname{reg}^{\mathcal{X}+1}(M) \leq$ $\operatorname{reg}^{\mathcal{X} \cup(\mathcal{X}+1)}(M / l M)-D+1=\operatorname{reg}(M / l M)-D+1$. Thus we get $\operatorname{reg}(M) \leq$ $\max \left\{\max H_{R_{+}}^{0}(M), \operatorname{reg}(M / l M)-D+1\right\}$. On the other hand, $\max H_{R_{+}}^{0}(M) \leq$ $\operatorname{reg}(M)$ and, by Proposition 4.5 (1), we have $\operatorname{reg}^{\mathcal{X}}(M / l M)-D+1 \leq \operatorname{reg}^{\mathcal{X} \cup(\mathcal{X}+1)}(M)$ $=\operatorname{reg}(M)$.

Theorem 4.8. Let $M$ be a finitely generated graded $R$-module with $\operatorname{dim}(M)=d$. Then

$$
\operatorname{reg}(M)=\max _{i \in\{0, \ldots, d\}}\left\{\alpha\left(M /\left(l_{1}, \ldots, l_{i}\right) M\right)-\sum_{j=1}^{i}\left(D_{j}-1\right)\right\},
$$

where $l_{1}, \ldots, l_{d}$ is a filter regular sequence of degrees $D_{1}, \ldots, D_{d}$.

Proof. By definition, given any finitely generated graded $R$-module $N$ and any $i>\operatorname{reg}(N)$, we have $H_{R_{+}}^{j}(N)_{i-j}=0$. In particular $H_{R_{+}}^{j}(N)_{i}=0$, hence from (4.2.1) it is clear that $\operatorname{reg}(N) \geq \alpha(N)$ for every $N$.

By Corollary 4.7. $\operatorname{reg}(M) \geq \operatorname{reg}(M / l M)-\operatorname{deg} l+1$ for any filter regular element $l$, so in particular we have

$$
\operatorname{reg}(M) \geq \max _{i \in\{0, \ldots, d\}}\left\{\alpha\left(M /\left(l_{1}, \ldots, l_{i}\right) M\right)-\sum_{j=1}^{i}\left(D_{j}-1\right)\right\} .
$$

We need to prove the reverse inequality. We do an induction on the dimension of $M$. If $\operatorname{dim} M=0$, then $\operatorname{reg}(M)=\max H_{R_{+}}^{0}(M)$ which equals to $\alpha(M)$, by (4.2.1). Assume $d>0$. By induction hypothesis we get

$$
\operatorname{reg}\left(M / l_{1} M\right)=\max _{i \in\{1, \ldots, d\}}\left\{\alpha\left(M /\left(l_{1}, l_{2}, \ldots, l_{i}\right) M\right)-\sum_{j=2}^{i}\left(D_{j}-1\right)\right\} .
$$

Consequently setting $a=\max _{i \in\{0, \ldots, d\}}\left\{\alpha\left(M /\left(l_{1}, \ldots, l_{i}\right) M\right)-\sum_{j=1}^{i}\left(D_{j}-1\right)\right\}$, we have

$$
\operatorname{reg}\left(M / l_{1} M\right)-D_{1}+1 \leq a
$$


Thanks to Corollary 4.7 we still have to prove that $\max H_{R_{+}}^{0}(M) \leq a$. Since $H_{R_{+}}^{j}(M) \cong H_{R_{+}}^{j}\left(M / H_{R_{+}}^{0}(M)\right)$ for all $j>0$, by Corollary 4.6 we know that $H_{R_{+}}^{j}(M)_{>a-j}=0$ for all $j>0$. In particular for any $b>a, H_{R_{+}}^{j}(M)_{b}=0$ for all $j>0$. Hence by (4.2.1) we have $H_{M}(b)-P_{M}(b)=\operatorname{dim}_{K} H_{R_{+}}^{0}(M)_{b}$. But $a \geq \alpha(M)$, so $H_{M}(b)-P_{M}(b)=0$ for all $b>a \geq \alpha(M)$. Therefore $\max H_{R_{+}}^{0}(M) \leq a$.

An interesting corollary of Theorem 4.8 is the following.

Corollary 4.9. Let $M$ be a finitely generated graded $R$-module such that $\operatorname{dim} M=$ $d$, and let $l_{1}, \ldots, l_{d}$ be a filter regular sequence on $M$ of elements of degree $D_{1}, \ldots, D_{d}$. Then the number

$$
\max _{i \in\{0, \ldots, d\}}\left\{\alpha\left(M /\left(l_{1}, \ldots, l_{i}\right) M\right)-\sum_{j=1}^{i}\left(D_{j}-1\right)\right\}
$$

is independent of the choice of the filter regular sequence and of its degrees.

Remark 4.10. Note that both Theorem 4.8 and Corollary 4.9 lie on the definition of $\alpha(M)$. The number $\alpha(M)$ is the highest integer $i$ for which the function $\phi$ defined as

$$
\phi\left(i, M_{0}, M_{1}, M_{2}, \ldots, M_{n}\right):=\sum_{j=0}^{n}(-1)^{j} \operatorname{dim}_{K}\left(M_{j}\right)_{i}
$$

is not zero at $\left(i, H_{R_{+}}^{0}(M), H_{R_{+}}^{1}(M), \ldots, H_{R_{+}}^{n}(M)\right)$. We want to point out that we can substitute for $\phi$ any other function $\psi$ such that, whenever $\left(M_{j}\right)_{>i-j}=0$ for all $j>0$, we have

$$
\psi\left(i, M_{0}, M_{1}, M_{2}, \ldots, M_{n}\right) \neq 0 \text { if and only if }\left(M_{0}\right)_{i} \neq 0 .
$$

Then instead of $\alpha(M)$ we could use the function $\beta(M)$ defined as

$$
\sup \left\{i \mid \psi\left(i, H_{R_{+}}^{0}(M), H_{R_{+}}^{1}(M), \ldots, H_{R_{+}}^{n}(M)\right) \neq 0\right\} .
$$

If we take, for example, $\operatorname{dim}_{K}\left(\left(M_{0}\right)_{i}\right)$ as a function of $\psi\left(i, M_{0}, \ldots, M_{n}\right)$, we obtain, as an analogue of Theorem 4.8, the following already known fact.

Theorem 4.11. Given a finitely generated module $M$ of dimension $d$, we have

$$
\operatorname{reg}(M)=\max _{i \in\{0, \ldots, d\}}\left\{\operatorname{sat}\left(M /\left(l_{1}, \ldots, l_{i}\right) M\right)-\sum_{j=1}^{i}\left(D_{j}-1\right)\right\},
$$

where $\operatorname{sat}(P)$ is defined to be $\max H_{R_{+}}^{0}(P)$.

Note that Theorem 4.11 can be found in $\mathrm{Gr}$ (see Theorem $2.30(5),(6))$ under the more restricted assumptions that the field $K$ has characteristic zero and the $l_{i}$ 's are generic linear forms. It can also be easily derived from $[\mathrm{CH}]$, Proposition 1.2.

\section{ACKNOWLEDGMENT}

I thank Professor Craig Huneke for many helpful comments concerning this paper. 


\section{REFERENCES}

[Au] M. Auslander, Modules over unramified regular local rings. Illinois J. Math. 5, 1961, 631647. MR0179211 (31:3460)

$[\mathrm{BM}]$ D. Bayer and D. Mumford, What can be computed in algebraic geometry? Computational algebraic geometry and commutative algebra (Cortona, 1991), 1-48, Sympos. Math., XXXIV, Cambridge Univ. Press, Cambridge, 1993. MR1253986 (95d:13032)

[BH] W. Bruns and J. Herzog, Cohen-Macaulay rings. Cambridge Studies in Advanced Mathematics, 39. Cambridge University Press, Cambridge, 1993. xii+403 pp. MR 1251956 (95h:13020)

[Ch] K. Chandler, Regularity of the powers of an ideal. Comm. Algebra 25 (1997), no. 12, 3773-3776. MR1481564 (98i:13040)

$[\mathrm{CH}]$ A. Conca and J. Herzog, Castelnuovo-Mumford regularity of products of ideals. Collect. Math. 54 (2003), no. 2, 137-152. MR1995137 (2004k:13020)

[GGP] A. Geramita, A. Gimigliano, and Y. Pitteloud, Graded Betti numbers of some embedded rational $n$-folds. Math. Ann. 301 (1995), no. 2, 363-380. MR.1314592 (96f:13022)

[Gi] D. Giaimo, Regularity of connected curves. In preparation (2003).

[Gr] M. Green, Generic initial ideals. Six lectures on commutative algebra. Papers from the Summer School on Commutative Algebra held in Bellaterra, July 16-26, 1996. Progress in Mathematics, 166, 119-186. MR1648665 (99m:13040)

[Ma] B. Malgrange, Cartan involutiveness = Mumford regularity. Commutative algebra (Grenoble/Lyon, 2001), 193-205, Contemp. Math. 331, Amer. Math. Soc., Providence, RI, 2003. MR2013167 (2005b:12013)

$[\mathrm{Mu}]$ D. Mumford, Lectures on curves on an algebraic surface. With a section by G. M. Bergman. Annals of Mathematics Studies, No. 59 Princeton University Press, Princeton, N.J., 1966. $\mathrm{xi}+200$ pp. MR0209285 (35:187)

[Si] J. Sidman, On the Castelnuovo-Mumford regularity of products of ideal sheaves. Adv. Geom. 2 (2002), no. 3, 219-229. MR 1924756 (2003f:13021)

[St] B. Sturmfels, Four counterexamples in combinatorial algebraic geometry. J. Algebra 230 (2000), no. 1, 282-294. MR 1774768 (2001g:13047)

Department of Mathematics, University of California, Berkeley, 970 Evans Hall \#3840, Berkeley, California 94720-3840

E-mail address: caviglia@math.berkeley.edu 